\title{
Colistin Resistance in Acinetobacter baumannii MDR-ZJ06 Revealed by a Multiomics Approach
}

\begin{abstract}
Xiaoting Hua 1,2, Lilin Liu ${ }^{1,2}$, Youhong Fang ${ }^{3}$, Qiucheng Shi ${ }^{1,2}, X_{i}$ Li $^{4}$, Qiong Chen ${ }^{5}$, Keren Shi ${ }^{1,2}$, Yan Jiang ${ }^{1,2}$, Hua Zhou ${ }^{6}$ and Yunsong Yu ${ }^{1,2,7 *}$

${ }^{1}$ Department of Infectious Diseases, Sir Run Run Shaw Hospital, College of Medicine, Zhejiang University, Hangzhou, China, ${ }^{2}$ Key Laboratory of Microbial Technology and Bioinformatics of Zhejiang Province, Hangzhou, China, ${ }^{3}$ The Children's Hospital, College of Medicine, Zhejiang University, Hangzhou, China, ${ }^{4}$ Centre of Laboratory Medicine, Zhejiang Provincial People's Hospital, Hangzhou, China, ${ }^{5}$ Hangzhou First People's Hospital, Hangzhou, China, ${ }^{6}$ Department of Respiratory, The First Affiliated Hospital, College of Medicine, Zhejiang University, Hangzhou, China, ${ }^{7}$ State Key Laboratory for Diagnosis and Treatment of Infectious Diseases, Collaborative Innovation Center for Diagnosis and Treatment of Infectious Diseases, The First Affiliated Hospital, College of Medicine, Zhejiang University, Hangzhou, China
\end{abstract}

Acinetobacter baumannii has emerged as an important opportunistic pathogen due to its ability to acquire resistance to most currently available antibiotics. Colistin is often considered as the last line of therapy for infections caused by multidrug-resistant A. baumannii (MDRAB). However, colistin-resistant $A$. baumannii strain has recently been reported. To explore how multiple drug-resistant $A$. baumannii responded to colistin resistance, we compared the genomic, transcriptional and proteomic profile of A. baumannii MDR-ZJ06 to the induced colistin-resistant strain ZJ06-200P5-1. Genomic analysis showed that IpxC was inactivated by ISAba1 insertion, leading to LPS loss. Transcriptional analysis demonstrated that the colistin-resistant strain regulated its metabolism. Proteomic analysis suggested increased expression of the RND efflux pump system and down-regulation of FabZ and $\beta$-lactamase. These alterations were believed to be response to LPS loss. In summary, the IpxC mutation not only established colistin resistance but also altered global gene expression.

\section{Keywords: Acinetobacter baumannii, colistin, whole-genome sequencing, transcriptome, proteome}

\section{INTRODUCTION}

Acinetobacter baumannii has emerged as an important opportunistic pathogen due to its ability to acquire resistance to most currently available antibiotics (Peleg et al., 2008; Howard et al., 2012; Antunes et al., 2014). Since current treatment options for multi-drug resistant (MDR) A. baumannii are extremely limited, colistin is often considered as the last line of the therapy for infections caused by MDR A. baumannii (Bae et al., 2016; Cheah et al., 2016b). However, colistin-resistant A. baumannii strain has recently been reported (Cai et al., 2012).

Colistin is a polycationic antimicrobial peptide that targets the polyanionic bacterial lipopolysaccharide (LPS) of Gram-negative bacteria. Two different colistin resistance mechanisms have previously been reported (Beceiro et al., 2014). The first mechanism inactivates the lipid A biosynthesis pathway, leading to the complete loss of surface LPS. Mutations in $l p x C, l_{p} x A$, or $l p \times D$ are involved in the first mechanism. The $p m r A B$ two-component system mediates the second resistance mechanism. Mutations in $p m r A$ and $p m r B$ induce the activity of $p m r C$, which adds 
phosphoethanolamine (PEtn) to the hepta-acylated form of lipid A (Beceiro et al., 2011). Further mutations in vacJ, pldA, ttg2C, pheS and a conserved hypothetical protein were reported to involve in reduced colistin susceptibility through novel resistance mechanisms (Thi Khanh Nhu et al., 2016). Four putative colistin resistant genes: A1S_1983, hepA, A1S_3026, and $r s f S$ were also identified in our previous study ( $\mathrm{Mu}$ et al., 2016).

The response to LPS alteration has been investigated via transcriptional analysis. In response to LPS alteration, A. baumannii alters the expression of critical transport and biosynthesis systems associated with modulating the composition and structure of the bacterial surface ( $l_{p} x A$; Henry et al., 2012) or alters the expression of genes associated with outer membrane structure and biogenesis ( $p m r B$; Cheah et al., 2016a). Moreover, the response to colistin is highly similar to the transcriptional alteration observed in an LPSdeficient strain (Henry et al., 2015). Colistin resistance was also explored using proteomic methods. There were 35 differentially expressed proteins. Most differentially expressed proteins were down-regulated in the colistin resistant strain, including outer membrane proteins, chaperones, protein biosynthesis factors, and metabolic enzymes (Fernandez-Reyes et al., 2009). However, the combination of genomic, transcriptomic, and proteomic methods to examine the colistin resistance mechanism in A. baumannii has rarely been reported. Furthermore, the strain used in this study was an MDR strain, but not laboratory strains (ATCC 19606, ATCC 17978) that do not represent clonal lineages in a clinical environment. Here, we used genome, transcriptome, and proteome to elucidate the colistin resistance mechanism in MDR A. baumannii. There was an ISAbal insertion in $\operatorname{lp} x C$ (ABZJ_03720) in ZJ06200P5-1 compared with the genome sequence of MDR-ZJ06,

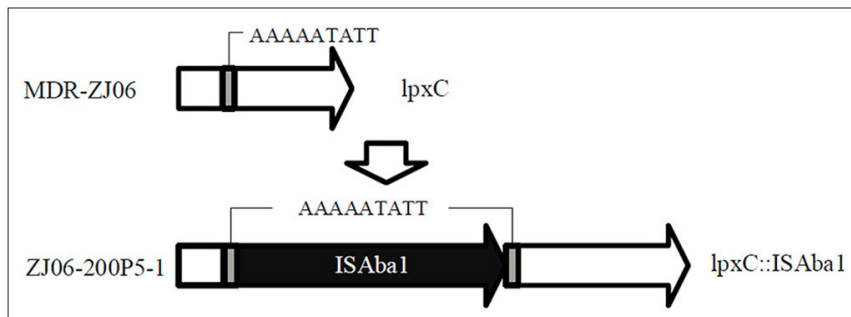

FIGURE 1 | Whole genome sequencing revealed the colistin-resistance mechanism in A. baumannii ZJ06-200P5-1. The gene IpxC was intact in MDR-ZJ06, while in ZJ06-200P5-1, IpXC was inactivated by the insertion sequence ISAba1 where $l p x C$ encoded an UDP-3-O-acyl-N-acetylglucosamine deacetylase.

\section{MATERIALS AND METHODS}

\section{Bacterial Strains, Media, and Antibiotics}

Restriction enzymes, T4 ligase, and Taq DNA polymerase were purchased from TaKaRa (Otsu, Shiga, Japan). The $A$. baumannii strain MDR-ZJ06 was isolated from the bloodstream of a patient in Hangzhou, China, in 2006. All A. baumannii cultures were grown at $37^{\circ} \mathrm{C}$ in Mueller-Hinton $(\mathrm{MH})$ agar and cation-adjusted $\mathrm{MH}$ broth (CAMHB) (Oxoid, Basingstoke, UK). Colistin was purchased from Sigma (Shanghai, China).

\section{Generation of Colistin-Resistant Mutant}

A colistin-resistant mutant was generated in A. baumannii MDR-ZJ06 by a previously described method (Li et al., 2006). Briefly, first, MDR-ZJ06 was cultured in CAMHB containing colistin at $8 \times$ minimum inhibitory concentration (MIC). After overnight incubation, the culture was diluted 1:1000 with CAMHB containing colistin at $64 \times$ MIC and then incubated at $37{ }^{\circ} \mathrm{C}$ overnight. Finally, the culture was diluted 1:100 with CAMHB containing colistin at $200 \times$ MIC. After overnight incubation, the culture was plated on plates containing $10 \mu \mathrm{g}$ of colistin at an appropriate dilution, and then one of colistin resistant colonies was collected for further experiments and designated as ZJ06-200P5-1. MICs for colistin and tigecycline were determined by E-test (bioMérieux, France) on $\mathrm{MH}$ agar, and the antimicrobial activities of the other antimicrobial agents were detected by disk diffusion. The results were interpreted according to CLSI or EUCAST breakpoints.

\section{Whole Genome DNA Sequencing and Analysis}

ZJ06-200P5-1 cells were cultured from a single colony overnight at $37{ }^{\circ} \mathrm{C}$ in $\mathrm{MH}$ broth. The genomic DNA was extracted via a QIAamp DNA minikit (Qiagen, Valencia, CA) following the manufacturer's protocol. Agarose gel and a NanoDrop spectrophotometer were used to determine the quality and quantity of extracted genomic DNA. The 300 bp library for Illumina paired-end sequencing was constructed from 5 $\mu \mathrm{g}$ of genome DNA of ZJ06-200P5-1 by staff at Zhejiang Tianke (Hangzhou, China). Mapping and SNP detection were performed via Breseq (Deatherage and Barrick, 2014). The regions containing the detected SNPs were amplified by PCR. The PCR products were sent to Biosune (Biosune, Hangzhou, China) for Sanger sequencing.

TABLE 1 | Antibiotic susceptibility of $A$. baumannii MDR-ZJ06 and its colistin resistant mutant ZJ06-200P5-1.

\begin{tabular}{|c|c|c|c|c|c|c|c|c|c|c|c|c|c|c|c|c|c|c|}
\hline Strains & $\mathrm{co}^{\mathrm{a}}$ & TGCa & IPM & MEM & FEP & CAZ & CTX & ATM & PRL & TZP & SCF & SAM & $\mathrm{CN}$ & AK & TE & MH & CIP & CT \\
\hline MDR-ZJ06 & 0.38 mg/L & 4 mg/L & 8 & 8 & 6 & 6 & 6 & 6 & 6 & 6 & 16 & 10 & 6 & 6 & 6 & 10 & 6 & 14 \\
\hline ZJ06-200P5-1 & $>256 \mathrm{mg} / \mathrm{L}$ & $0.5 \mathrm{mg} / \mathrm{L}$ & 22 & 22 & 20 & 20 & 15 & 22 & 17 & 19 & 30 & 22 & 6 & 6 & 8 & 26 & 9 & 6 \\
\hline
\end{tabular}

CO, colistin; TGC, tigecycline; IPM, imipenem; MEM, meropenem; FEP, cefepime; CAZ, ceftazidime; CTX, cefotaxime; ATM, aztreonam; PRL, Piperacillin; TZP, piperacillin/tazobactam; SCF, Cefoperazone/sulbactam; SAM, ampicillin/sulbactam; CN, gentamicin; AK, amikacin; TE, tetracycline; MH, minocycline; CIP, Ciprofloxacin; CT, colistin.

${ }^{a}$ The MIC of colistin and tigecycline were determined by broth dilution method, while antimicrobial sensitivity of other antibiotics were detected by disk diffusion. 
TABLE 2 | Genes changed significantly in transcriptome.

\begin{tabular}{|c|c|c|c|c|c|}
\hline Synonym & Product & $\log \mathrm{FC}$ & $\log \mathrm{CPM}$ & $P$-value & FDR \\
\hline ABZJ_00055 & hypothetical protein & 8.308068 & 13.717 & $1.26 \mathrm{E}-78$ & 4.54E-76 \\
\hline ABZJ_00068 & hypothetical protein & 6.4468 & 9.203574 & $2.14 \mathrm{E}-67$ & 4.61E-65 \\
\hline ABZJ_00037 & hypothetical protein & 4.368832 & 9.669037 & $3.48 \mathrm{E}-68$ & 9.36E-66 \\
\hline ABZJ_00056 & hypothetical protein & 4.349519 & 12.2059 & $6.03 E-65$ & 1.08E-62 \\
\hline ABZJ_00332 & hypothetical protein & 4.264896 & 9.455077 & 2.39E-53 & 2.86E-51 \\
\hline ABZJ_01880 & putative transposase & 2.758133 & 6.676606 & $5.52 \mathrm{E}-27$ & 3.13E-25 \\
\hline ABZJ_01079 & hypothetical protein & 2.585295 & 6.001793 & 4.14E-10 & $6.55 \mathrm{E}-09$ \\
\hline ABZJ_03753 & hypothetical protein & 2.318997 & 9.492231 & $2.51 \mathrm{E}-21$ & 1.08E-19 \\
\hline ABZJ_00333 & hypothetical protein & 2.314205 & 5.437541 & 2.36E-11 & 4.53E-10 \\
\hline ABZJ_01881 & transposase component & 2.25458 & 8.338274 & $9.50 \mathrm{E}-21$ & 3.93E-19 \\
\hline ABZJ_00060 & Thiol-disulfide isomerase and thioredoxin & 1.894318 & 12.3252 & 7.68E-20 & $2.75 \mathrm{E}-18$ \\
\hline ABZJ_00894 & lactoylglutathione lyase-like protein & 1.797874 & 6.779815 & $5.27 \mathrm{E}-15$ & 1.62E-13 \\
\hline ABZJ_00054 & N-alpha-acetylglutamate synthase (amino-acid acetyltransferase) & 1.77044 & 10.25589 & 3.24E-20 & 1.27E-18 \\
\hline ABZJ_01151 & hypothetical protein & 1.634908 & 3.574211 & 4.88E-06 & 3.84E-05 \\
\hline ABZJ_03714 & hypothetical protein & 1.61859 & 8.500912 & 1.39E-08 & 1.85E-07 \\
\hline ABZJ_01900 & acetoin:2,6-dichlorophenolindophenol oxidoreductase subunit alpha & 1.527437 & 6.102611 & $2.98 \mathrm{E}-06$ & 2.49E-05 \\
\hline ABZJ_01222 & hypothetical protein & 1.515854 & 2.111384 & 0.011897 & 0.034227 \\
\hline ABZJ_01191 & hypothetical protein & 1.46809 & 2.203352 & 0.011349 & 0.032877 \\
\hline ABZJ_01872 & hypothetical protein & 1.423713 & 7.613403 & 1.64E-08 & 2.10E-07 \\
\hline ABZJ_01187 & hypothetical protein & 1.423595 & 5.112417 & 2.82E-07 & 2.81E-06 \\
\hline ABZJ_01857 & hypothetical protein & 1.411761 & 2.566001 & 0.010144 & 0.029905 \\
\hline ABZJ_00977 & hypothetical protein & 1.209422 & 3.887522 & 0.000232 & 0.001118 \\
\hline ABZJ_00102 & D-lactate dehydrogenase FAD-binding protein & 1.170013 & 8.813908 & $1.91 \mathrm{E}-10$ & 3.15E-09 \\
\hline ABZJ_01149 & hypothetical protein & 1.156232 & 3.314522 & 0.003302 & 0.011138 \\
\hline ABZJ_00053 & alkanesulfonate transport protein & 1.143156 & 6.421362 & 5.15E-06 & 3.99E-05 \\
\hline ABZJ_01275 & hypothetical protein & 1.122845 & 8.385252 & $1.31 \mathrm{E}-08$ & 1.76E-07 \\
\hline ABZJ_03838 & membrane-fusion protein & 1.119324 & 7.708838 & 1.84E-08 & 2.33E-07 \\
\hline ABZJ_01901 & acetoin:26-dichlorophenolindophenol oxidoreductase beta subunit & 1.105826 & 6.349341 & 5.58E-05 & 0.000323 \\
\hline ABZJ_01899 & lipoate synthase & 1.08338 & 4.583472 & 0.003397 & 0.011422 \\
\hline ABZJ_00360 & hypothetical protein & 1.076106 & 8.065171 & 1.34E-07 & 1.46E-06 \\
\hline ABZJ_01210 & hypothetical protein & 1.065917 & 3.456549 & 0.011028 & 0.032156 \\
\hline ABZJ_01160 & hypothetical protein & 1.048988 & 3.144467 & 0.012194 & 0.034895 \\
\hline ABZJ_01148 & hypothetical protein & 1.048966 & 5.540519 & 1.77E-05 & 0.000122 \\
\hline ABZJ_00099 & L-lactate permease & 1.044891 & 10.0835 & 8.49E-08 & $9.61 \mathrm{E}-07$ \\
\hline ABZJ_00901 & major facilitator superfamily multidrug resistance protein & 1.016944 & 9.235389 & $1.47 \mathrm{E}-08$ & $1.91 \mathrm{E}-07$ \\
\hline ABZJ_01775 & 6-pyruvoyl-tetrahydropterin synthase & 1.014549 & 10.17374 & $3.05 E-12$ & $6.84 \mathrm{E}-11$ \\
\hline ABZJ_03786 & VirP protein & -1.0004 & 6.133241 & 3.35E-06 & 2.73E-05 \\
\hline ABZJ_01269 & TPR repeat-containing SEL1 subfamily protein & -1.00222 & 4.702232 & 0.000305 & 0.001408 \\
\hline
\end{tabular}


TABLE 2 | Continued

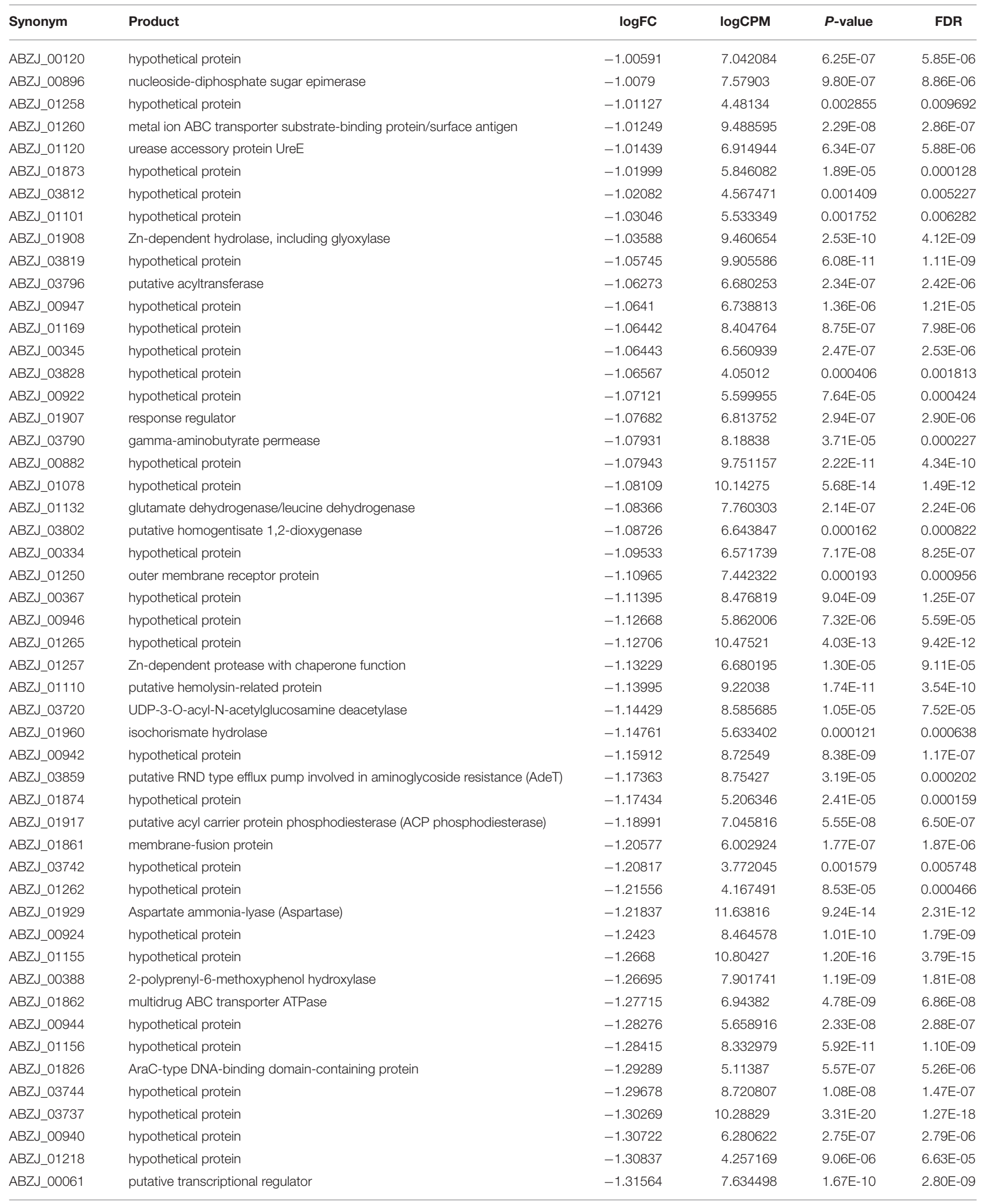


TABLE 2 | Continued

\begin{tabular}{|c|c|c|c|c|c|}
\hline Synonym & Product & $\log \mathrm{FC}$ & $\log C P M$ & $P$-value & FDR \\
\hline ABZJ_01887 & hypothetical protein & -1.3281 & 6.449578 & $1.02 \mathrm{E}-07$ & 1.14E-06 \\
\hline ABZJ_01025 & homocysteine/selenocysteine methylase & -1.33719 & 7.528478 & 3.07E-10 & 4.93E-09 \\
\hline ABZJ_00110 & GNAT family acetyltransferase & -1.33942 & 4.887691 & 1.06E-06 & 9.50E-06 \\
\hline ABZJ_01242 & hypothetical protein & -1.3506 & 7.369014 & 2.45E-09 & 3.61E-08 \\
\hline ABZJ_00895 & hypothetical protein & -1.35351 & 6.693904 & 7.37E-12 & $1.56 \mathrm{E}-10$ \\
\hline ABZJ_03712 & putative flavoprotein & -1.38598 & 6.6067 & 2.04E-09 & 3.04E-08 \\
\hline ABZJ_00048 & transcriptional regulator & -1.40027 & 7.755295 & $9.36 \mathrm{E}-11$ & 1.68E-09 \\
\hline ABZJ_03785 & glutamate racemase & -1.40496 & 7.417511 & 7.08E-12 & $1.52 \mathrm{E}-10$ \\
\hline ABZJ_00938 & hypothetical protein & -1.40799 & 6.629998 & 1.09E-10 & 1.88E-09 \\
\hline ABZJ_01230 & hypothetical protein & -1.41279 & 10.19585 & 3.47E-19 & 1.20E-17 \\
\hline ABZJ_00124 & glycine/D-amino acid oxidase (deaminating) & -1.46015 & 13.3987 & $8.58 \mathrm{E}-14$ & $2.20 \mathrm{E}-12$ \\
\hline ABZJ_03791 & histidine ammonia-lyase (Histidase) & -1.49736 & 9.748038 & 2.37E-08 & 2.90E-07 \\
\hline ABZJ_03739 & hypothetical protein & -1.49749 & 13.98113 & $3.54 \mathrm{E}-13$ & 8.47E-12 \\
\hline ABZJ_00881 & glutamine amidotransferase & -1.51327 & 8.144142 & 5.09E-14 & 1.37E-12 \\
\hline ABZJ_00988 & hypothetical protein & -1.54819 & 6.1324 & 7.44E-09 & 1.05E-07 \\
\hline ABZJ_01840 & putative ferric siderophore receptor protein & -1.55785 & 9.806018 & $9.74 \mathrm{E}-10$ & $1.52 \mathrm{E}-08$ \\
\hline ABZJ_00997 & hypothetical protein & -1.58106 & 5.257799 & $3.12 \mathrm{E}-08$ & 3.77E-07 \\
\hline ABZJ_00339 & HSP90 family molecular chaperone & -1.6168 & 11.15864 & 7.57E-23 & $3.54 \mathrm{E}-21$ \\
\hline ABZJ_00373 & Type II secretory pathway, ATPase PuIE/Tfp pilus assembly pathway, ATPase PilB & -1.6419 & 6.706339 & $3.45 \mathrm{E}-14$ & $9.79 \mathrm{E}-13$ \\
\hline ABZJ_01845 & phosphatase/phosphohexomutase & -1.68301 & 7.222507 & 3.67E-12 & 8.06E-11 \\
\hline ABZJ_03793 & urocanate hydratase & -1.69267 & 10.89217 & 1.13E-07 & 1.25E-06 \\
\hline ABZJ_03754 & Rhs element Vgr family protein & -1.69503 & 8.757228 & $5.86 \mathrm{E}-18$ & 1.97E-16 \\
\hline ABZJ_00945 & hypothetical protein & -1.72533 & 5.192791 & $2.02 \mathrm{E}-11$ & 4.03E-10 \\
\hline ABZJ_01002 & putative ABC oligo/dipeptide transport, ATP-binding protein & -1.73182 & 6.449009 & $4.32 \mathrm{E}-14$ & 1.19E-12 \\
\hline ABZJ_01259 & hypothetical protein & -1.75565 & 7.198513 & $1.30 \mathrm{E}-12$ & $2.98 \mathrm{E}-11$ \\
\hline ABZJ_00114 & short chain dehydrogenase family protein & -1.76754 & 7.176594 & 1.03E-13 & $2.52 \mathrm{E}-12$ \\
\hline ABZJ_01177 & hypothetical protein & -1.8053 & 8.135954 & $6.06 \mathrm{E}-15$ & $1.81 \mathrm{E}-13$ \\
\hline ABZJ_03792 & hypothetical protein & -1.82418 & 6.284478 & $3.56 \mathrm{E}-06$ & 2.88E-05 \\
\hline ABZJ_01219 & hypothetical protein & -1.86448 & 9.22858 & 7.68E-22 & $3.45 E-20$ \\
\hline ABZJ_01088 & carbonic anhydrase & -1.94984 & 9.430551 & $1.08 \mathrm{E}-27$ & 6.83E-26 \\
\hline ABZJ_00346 & hypothetical protein & -2.03948 & 6.219886 & $1.15 \mathrm{E}-16$ & $3.73 E-15$ \\
\hline ABZJ_01207 & hypothetical protein & -2.1746 & 7.126199 & $6.11 \mathrm{E}-20$ & $2.27 \mathrm{E}-18$ \\
\hline ABZJ_01886 & hypothetical protein & -2.33548 & 5.458495 & 1.05E-11 & $2.18 \mathrm{E}-10$ \\
\hline ABZJ_03766 & putative secretory lipase precursor & -2.38284 & 9.073946 & $1.11 \mathrm{E}-31$ & 7.47E-30 \\
\hline ABZJ_01206 & hypothetical protein & -3.28101 & 9.194837 & $2.48 \mathrm{E}-45$ & $2.42 \mathrm{E}-43$ \\
\hline ABZJ_03736 & thiol:disulfide interchange protein & -3.9361 & 9.872762 & $6.64 \mathrm{E}-41$ & 5.50E-39 \\
\hline
\end{tabular}

\section{Transcriptome Analysis and Real-Time Quantitative PCR Verification}

A. baumannii MDR-ZJ06 and ZJ06-200P5-1 were grown overnight at $37^{\circ} \mathrm{C}$ in LB broth. Strains were subcultured $1 / 100$ into fresh $\mathrm{LB}$ broth and grown at $37^{\circ} \mathrm{C}$ for $2 \mathrm{~h}\left(\mathrm{OD}_{600}: 0.29\right.$ \pm 0.02 for MDR-ZJ06, $0.26 \pm 0.02$ for ZJ06-200P5-1). The cells were collected at $4{ }^{\circ} \mathrm{C}$, and the RNA was extracted using TRIZOL Reagent (Invitrogen, Carlsbad, CA, USA) after liquid nitrogen grinding. For RNA sequencing, wild type and mutants were sampled in triplicate. The subsequent RNA extraction, bacteria mRNA sequence library construction, transcriptome analysis and real-time quantitative PCR verification were performed by staff at Zhejiang Tianke (Hangzhou, China) as described previously in reference (Hua et al., 2014). Sequenced reads were mapped to the MDR-ZJ06 genome (CP001937-8) using Rockhopper
(McClure et al., 2013). The output data was analyzed by edgeR (McCarthy et al., 2012). Data generated by RNA sequencing were deposited to the NCBI Sequence Read Archive with accession number SRR5234544 (the wild type) and SRR5234545 (the colistin resistant strain).

\section{Proteomic Analysis}

A. baumannii MDR-ZJ06 and ZJ06-200P5-1 were grown overnight at $37^{\circ} \mathrm{C}$ in LB broth. Strains were subcultured $1 / 100$ into fresh $\mathrm{LB}$ broth and grown at $37^{\circ} \mathrm{C}$ for $2 \mathrm{~h}\left(\mathrm{OD}_{600}: 0.29\right.$ \pm 0.02 for MDR-ZJ06, $0.26 \pm 0.02$ for ZJ06-200P5-1). The cells were collected at $4{ }^{\circ} \mathrm{C}$ and sent to Shanghai Applied Protein Technology Co. Ltd. The cell pellets were washed twice with PBS, and $500 \mu \mathrm{l} \mathrm{SDT}$ lysis buffer (4\% SDS, $100 \mathrm{mM}$ Tris- $\mathrm{HCl}, 1 \mathrm{mM}$ DTT, pH 7.6) was added. After being sonicated for 2 mins on ice, 


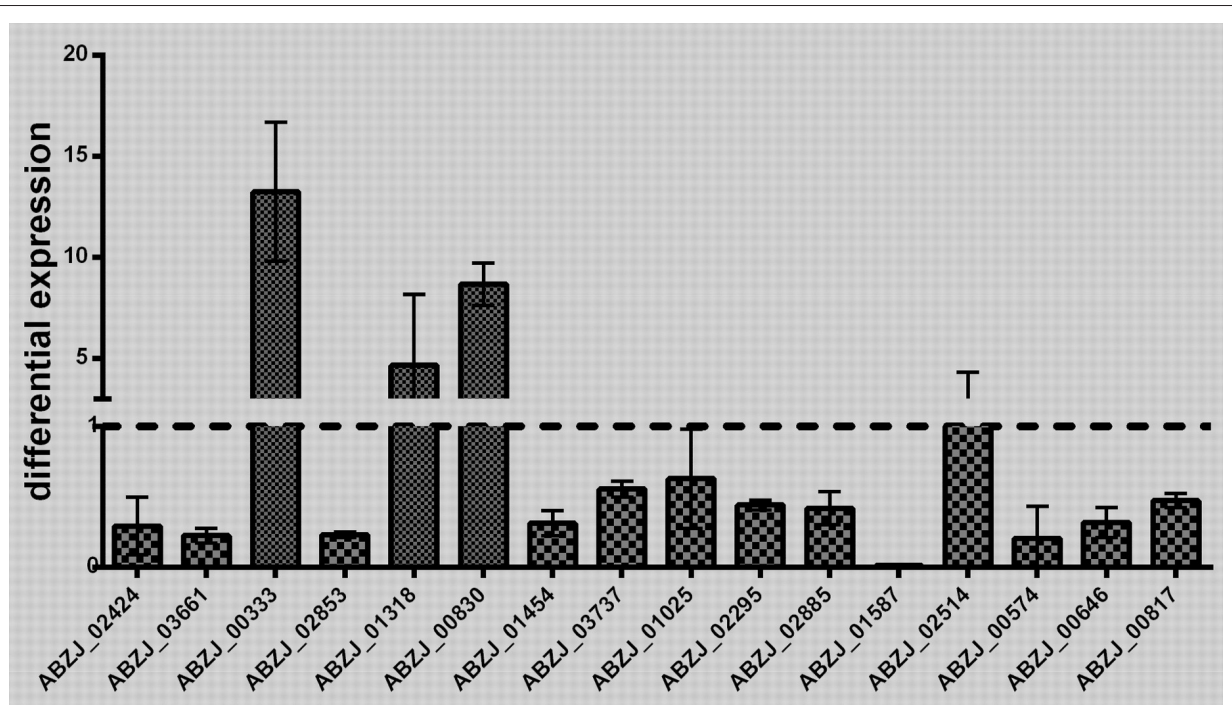

FIGURE 2 | Validation of the RNA sequencing results. The transcriptomic results obtained by RNA-seq were validated by quantitative RT-PCR analysis. The differential expression of 16 genes was detected in this study. Three biology replicates were used in this experiment. The results were presented as expression in ZJ06-200P5-1, relative to MDR-ZJ06. The reference gene rpoB was used for inter-sample normalization. Error bars denote standard deviation.

the cells were centrifuged at $14,000 \times \mathrm{g}$ for $30 \mathrm{~min}$ at $4^{\circ} \mathrm{C}$. The protein concentration in the supernatant was determined by the BCA method.

In brief, $300 \mu \mathrm{g}$ protein was added to $200 \mu \mathrm{l} \mathrm{UA}$ buffer $(8 \mathrm{M}$ urea, $150 \mathrm{mM}$ Tris- $\mathrm{HCl} \mathrm{pH}$ 8.0) and ultrafiltered (Sartorius, $10 \mathrm{kD}$ ) with UA buffer. To block reduced cysteine residues, $100 \mu$ l iodoacetamide (IAA) buffer (50 mM IAA in UA buffer) was added, centrifuged at $600 \mathrm{rpm}$ for $1 \mathrm{~min}$, and incubated for $30 \mathrm{~min}$ in the dark. The filter was washed twice with $100 \mu \mathrm{l}$ UA buffer and twice with $100 \mu \mathrm{l}$ Dissolution buffer (50 $\mathrm{mM}$ triethylammonium bicarbonate, $\mathrm{pH} 8.5$ ). Finally, the proteins were digested with $2 \mu \mathrm{g}$ trypsin (Promega) in 40 $\mu \mathrm{l}$ Dissolution buffer at $37{ }^{\circ} \mathrm{C}$ for $16-18 \mathrm{~h}$. The peptides were collected as a filtrate, and its content was estimated at $\mathrm{OD}_{280}$.

For iTRAQ labeling, the peptides were labeled with the 4-plex iTRAQ reagent following the manufacturer's instructions (AB SCIEX). The peptides from MDR-ZJ06 were labeled with 114 and 116 isobaric reagents, and the peptides from ZJ06-200P5-1 were labeled with 115 and 117 isobaric reagents.

RP-HPCL online-coupled to MS/MS (LC-MS/MS) analysis of the iTRAQ-labeled peptides was performed on an EASYnLC nanoflow LC system (Thermo Fisher Scientific) connected to an Orbitrap Elite hybrid mass spectrometer (Thermo Fisher Scientific). After the samples were reconstituted and acidified with buffer $\mathrm{A}(0.1 \%(\mathrm{v} / \mathrm{v})$ formic acid in water), a set-up involving a pre-column and analytical column was used. The pre-column was a $2 \mathrm{~cm}$ EASY-column (100, $5 \mu \mathrm{m}$ C18; Thermo Fisher Scientific), while the analytical column was a $10 \mathrm{~cm}$ EASYcolumn (75, $3 \mu \mathrm{m}, \mathrm{C} 18$; Thermo Fisher Scientific). The 120 min linear gradient from 0 to $100 \%$ buffer B $(0.1 \%$ (v/v) formic acid and $80 \%$ acetonitrile) at a constant flow rate of $250 \mathrm{nl} / \mathrm{min}$ was as follows: $0-100 \mathrm{~min}, 0-35 \%$ buffer B; 100-108 $\mathrm{min}, 35-$ $100 \%$ buffer B; 108-120 min, $100 \%$ buffer B. MS data were acquired using a data-dependent top 10 method, dynamically choosing the most abundant precursor ions from the survey scan $(300-180 \mathrm{~m} / \mathrm{z})$ for HCD fragmentation. The Dynamic exclusion was set to a repeat count of 1 with a $30 \mathrm{~s}$ duration. Survey scans were acquired at a resolution of 30,000 at $\mathrm{m} / \mathrm{z} \mathrm{200}$, and the resolution for HCD spectra was set to 15,000 at $\mathrm{m} / \mathrm{z} 200$. The normalized collision energy was $35 \mathrm{eV}$, and the underfill ratio was defined as $0.1 \%$.

The MS/MS spectra were searched using the MASCOT engine (Matrix Science, London, UK; version 2.2) against the A. baumannii MDR-ZJ06 FASTA database. False discovery rates (FDR) were calculated via running all spectra against the FASTA database using the MASCOT software. The following options were used to identify proteins: peptide mass tolerance $=20 \mathrm{ppm}$, fragment mass tolerance $=0.1$ Da, Enzyme $=$ Trypsin, Max missed cleavages $=2$, Fixed modification: Carbamidomethyl (C), iTRAQ 4plex (N-term), iTRAQ 4plex (K), Variable modification: Oxidation (M). Quantification was performed based on the peak intensities of the reporter ions in the MS/MS spectra. The proteins were considered overexpressed when the iTRAQ ratio was above 1.5 and underexpressed when the iTRAQ ratio was lower than 0.67 (Wang et al., 2016). Functional classification of differentially expression genes were annotated using the KEGG databases. The mass spectrometry proteomics data have been deposited to the ProteomeXchange Consortium via the PRIDE (Vizcaino et al., 2016) partner repository with the dataset identifier PXD005265 and 10.6019/PXD005265. Reviewer account details: Username: reviewer54242@ebi.ac.uk; Password: zR8mE9wu.

\section{Growth Rate Determination}

Four independent cultures per strain were grown overnight, diluted to 1:1000 in $\mathrm{MH}$ and aliquots placed into a flat-bottom 100 -well plate in four replicates. The plate was incubated at $37^{\circ} \mathrm{C}$ 
with agitation. The $\mathrm{OD}_{600}$ of each culture was determined every 5 min for 16 h using a Bioscreen C MBR machine (Oy Growth Curves Ab Ltd., Finland). The growth rate was estimated based on $\mathrm{OD}_{600}$ curves using an R script (Fang et al., 2016).

\section{RESULTS}

\section{Whole Genome Sequencing, Minimum Inhibitory Concentration and Growth Rate}

The colistin-resistant mutant ZJ06-200P5-1 generated from the culture in CAMHB containing colistin was sent for whole genome sequencing. There was an ISAbal insertion in $\operatorname{lp} x \mathrm{C}$ in ZJ06-200P5-1 compared with the genome sequence of MDRZJ06 (Figure 1). The MIC of MDR-ZJ06 and ZJ06-200P5-1 were detected and listed in Table 1. The MIC for colistin increased from $0.38 \mathrm{mg} / \mathrm{L}$ (MDR-ZJ06) to >256 mg/L (ZJ06200P5-1). However, ZJ06-200P5-1 showed higher sensitivity to multiple antibiotics: $\beta$-lactams, carbapenem, tetracycline, and ciprofloxacin, but not aminoglycosides. Furthermore, ZJ06200P5-1 showed a lower growth rate $(0.81 \pm 0.05)$ than wild type.

\section{Transcriptome Analysis}

The transcriptome analysis of ZJ06-200P5-1 and MDR-ZJ06 was performed by Illumina RNA deep sequencing technology. Cells of the two strains were collected in the early exponential phase. A total of 137 genes showed significant differential expression [ $\log 2$ (FoldChange) $>1$ or $\log 2$ (FoldChange) $<$ -1 ], among which 48 genes were upregulated and 89 were downregulated (Table 2). Sixteen selected genes, three upregulated and thirteen down-regulated genes, were well-validated by RT-qPCR (Figure 2). After mapping the differentially expressed genes into the KEGG pathway, we observed that genes involved in Energy metabolism and Amino acid metabolism were down-regulated, while Carbohydrate metabolism was up-regulated.

\section{ITRAQ}

A total of 1582 proteins were identified in the iTRAQ experiment. A protein ratio $>1.5$ or $<0.67(p<0.05)$ was considered to be differentially expressed. After filtration, 82 differentially expressed proteins were identified between ZJ06-200P5-1 and MDR-ZJ06. The detailed information is shown in Table 3.

The expression of AdeABC was up-regulated in the LPSloss ZJ06-200P5-1 strain. The AdeABC efflux pump confers resistance to various antibiotics classes. The expression of AdeABC genes was increased approximately two-fold in ZJ06-200P5-1 (Figure 3A). However, ZJ06-200P5-1 showed higher susceptibility to multiple antibiotics than MDR-ZJ06 (Table 1).

The fatty acid biosynthesis pathway was down-regulated in the ZJ06-200P5-1 strain (Figure 3B). The expression of FabZ was decreased by approximately two-fold in ZJ06-200P5-1. The $\beta$-lactamases $b l a_{\mathrm{OXA}-23}$ and $b l a_{\mathrm{ADC}-25}$ were down-regulated in ZJ06-200P5-1 strain. The expression levels of bla OXA-23 and bla $a_{\mathrm{ADC}-25}$ were decreased two- to four-fold in ZJ06200P5-1.

\section{Common Genes Altered Expression in Both Transcriptome and Proteome}

A total of 15 differentially expressed genes (or proteins) were identified in both transcriptome and proteome (Table 4). Among them, three genes were both up-regulated, and nine genes were both down-regulated. Although there was correlation between transcriptome and proteome data, the absolute expression difference values in transcriptome data was higher than those in proteome data. In addition, the result of three gene/proteins were contradictory (highlighted in red letters in Table 4). The contradictory result might be caused by post-transcriptional regulation.

\section{DISCUSSION}

Due to the limitation of antimicrobial agents in clinical use, it is urgent to extend our understanding of the emergence of colistin resistance in A. baumannii. A. baumannii MDR-ZJ06, a multidrug-resistant clinical strain isolated from bloodstream, has been sequenced and was considered an ideal strain for examining the colistin-resistant mechanism in A. baumannii (Zhou et al., 2011). In this study, colistin-resistant strain was rapidly obtained, and its resistance mechanism was LPS loss caused by ISAba1 insertion in $l_{p x C}$. This result confirmed a previous finding (Moffatt et al., 2010). The rapid isolation of colistin-resistant mutant from multiple drug-resistant A. baumannii indicated a high risk of $A$. baumannii evolving resistance to colistin in clinical use.

We successfully detected the whole transcriptional profile of A. baumannii strain MDR-ZJ06 and its colistinresistant mutant ZJ06-200P5-1 via Illumina RNA-sequencing. In another transcriptome study (Henry et al., 2012), A. baumannii ATCC 19606 and its $l p x A$ mutant were used. Although both the $\operatorname{lp} x C$ and $l p x A$ mutation lead to LPS loss, the different transcriptional response may be due to differences in the strain genetic background and the resistant mutation. In transcriptional analysis, we observed that genes involved in Energy metabolism and Amino acid metabolism were down-regulated, while Carbohydrate metabolism was up-regulated.

The expression of AdeABC was up-regulated in the LPS-loss ZJ06-200P5-1 strain. Similar results were also observed in all polymyxin-treated samples (Cheah et al., 2016a). In addition, the expression levels of adeIJK and macAB-tolC were up-regulated in the LPS loss mutant (Henry et al., 2012). Increased expression of the RND efflux pump system (AdeABC) was a common finding across all experiments in colistin exposure. The up-regulation of AdeABC indicated the diminished integrity and barrier function of the outer membrane in colistin-resistant A. baumannii (Henry et al., 2015; Cheah et al., 2016a). However, ZJ06200P5-1 showed higher susceptibility to multiple antibiotics than MDR-ZJ06. The higher susceptibility might result from the higher outer membrane permeability of ZJ06-200P5-1 due 


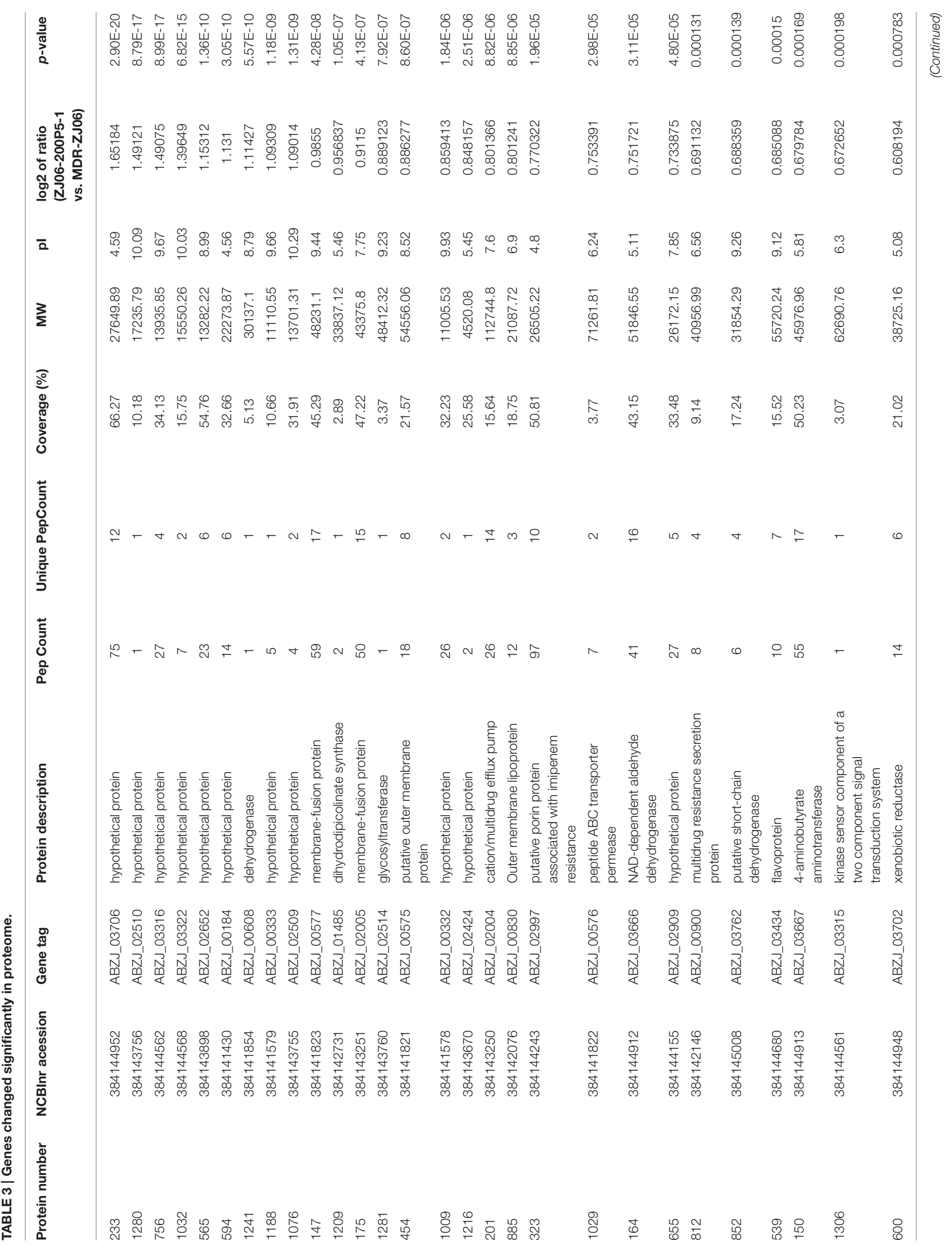




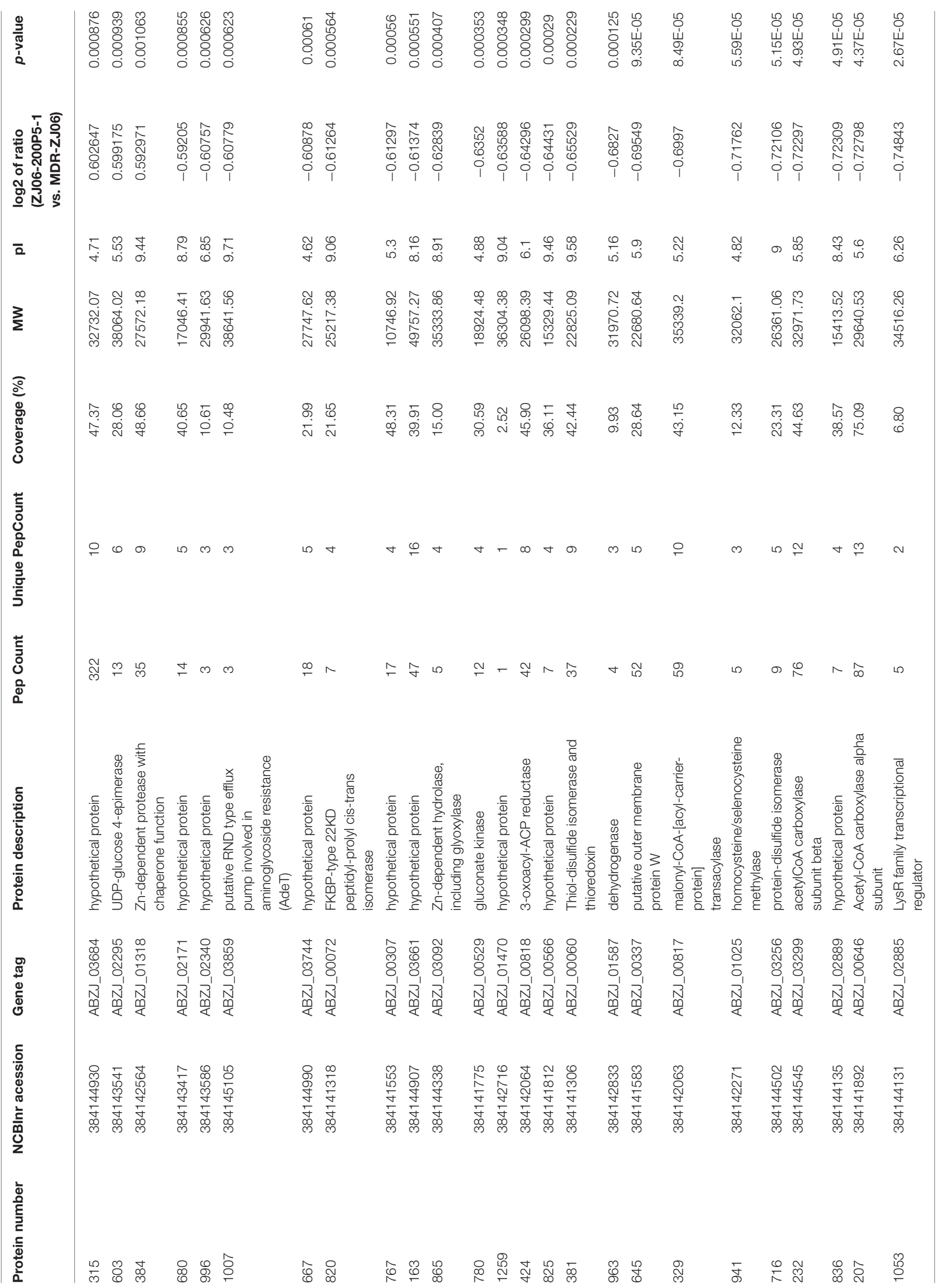




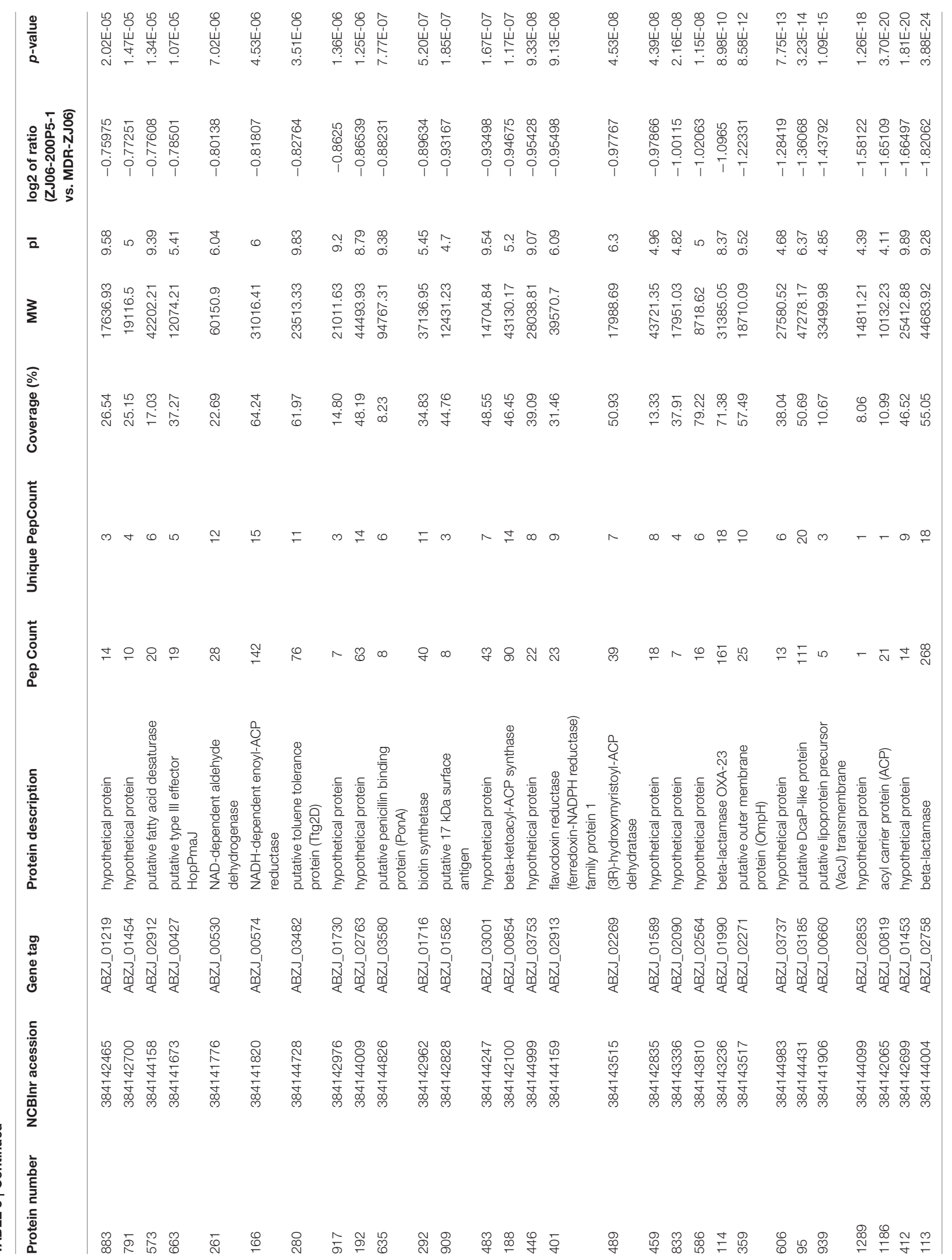


A

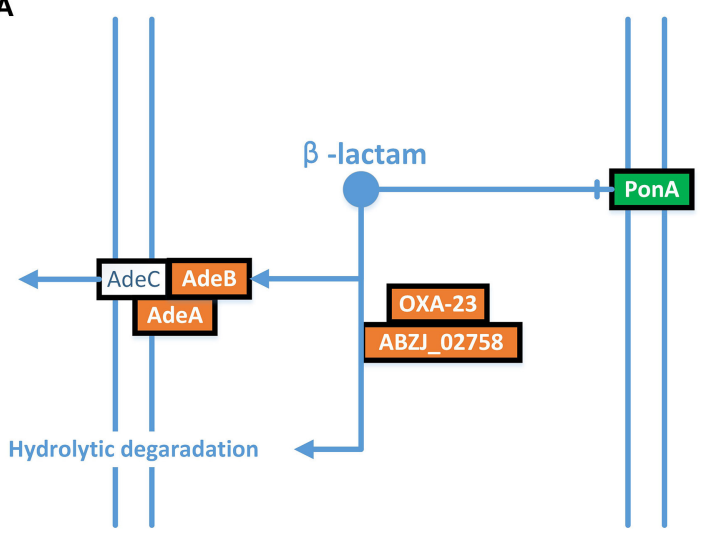

B

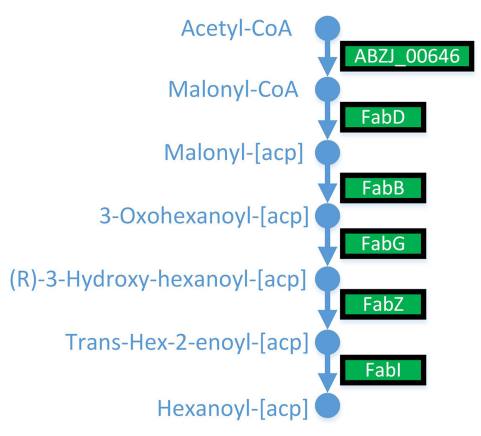

FIGURE 3 | ITRAQ analysis showed that AdeABC were up-regulated, and the fatty acid biosynthesis pathway was down-regulated in ZJ06-200P5-1. (A) AdeABC efflux pump, (B) fatty acid biosynthesis pathway. Green shows genes with significantly reduced expression levels, and red shows genes with significantly increased expression levels.

to LPS-loss. The increased expression of the efflux pump was thought to be a response to toxic substances that accumulated in the cells due to the increased membrane permeability (Henry et al., 2012).

The fatty acid biosynthesis pathway was down-regulated in the ZJ06-200P5-1 strain. In E. coli, it is important to balance LPS and fatty acid biosynthesis to maintain cell integrity. FabZ, which dehydrates R-3-hydroxymyristoyl-acyl carrier protein in fatty acid biosynthesis, plays an important role in rebalancing lipid A and fatty acid homeostasis (Bojkovic et al., 2016). The decrease in FabZ was considered to be a response to LPS-loss in ZJ06-200P5-1. The $\beta$-lactamases bla $a_{\mathrm{OXA}-23}$ and bla $a_{\mathrm{ADC}-25}$ were down-regulated in the ZJ06-200P5-1 strain. Decreased expression levels of bla $a_{\mathrm{OXA}-23}$ and $b l a_{\mathrm{ADC}-25}$ were also observed in A. baumannii MDR-ZJ06 under a subinhibitory concentration of tigecycline (Hua et al., 2014). Meanwhile, the strain under tigecycline stress showed a lower MIC of ceftazidime (Hua et al., 2014). The decrease in bla $a_{\mathrm{OXA}-23}$ and $b l a_{\mathrm{ADC}-25}$ might contribute to the increased sensitivity to $\beta$-lactam antimicrobial agents.

A multi-omics approach was adopted to obtain a more global view of colistin-resistant A. baumannii. Genomic analysis showed that $l p x C$ was inactivated by ISAbal insertion, leading to LPS loss. Transcriptional analysis demonstrated that the colistinresistant strain regulated its metabolism. Metabolic change and LPS loss were concomitant. Proteomic analysis suggested increased expression of the RND efflux pump system and the down-regulation of FabZ and $\beta$-lactamase. These alterations are believed to be responses to LPS loss. Together, the $l p x C$ mutation not only confirmed colistin resistance but also altered global gene expression.

\section{Nucleotide Sequence Accession Numbers}

The whole-genome shotgun sequencing results for A. baumannii ZJ06-200P5-1 have been deposited at DDBJ/EMBL/GenBank under the accession number MIFW00000000.
TABLE 4 | Common genes altered expression both in transcriptome and proteome.

\begin{tabular}{|c|c|c|c|}
\hline Synonym & Product & $\begin{array}{c}\text { Fold change } \\
\text { (log2, Transcriptome) }\end{array}$ & $\begin{array}{c}\text { Fold change } \\
\text { (log2, Proteome) }\end{array}$ \\
\hline ABZJ_00332 & hypothetical protein & 4.26489563 & 0.859413 \\
\hline ABZJ_03753 & hypothetical protein & $2.318997325^{a}$ & -0.95428 \\
\hline ABZJ_00333 & hypothetical protein & 2.314204886 & 1.09309 \\
\hline ABZJ_01133 & heat shock protein & 2.180888936 & 0.532117 \\
\hline ABZJ_00060 & $\begin{array}{l}\text { Thiol-disulfide } \\
\text { isomerase and } \\
\text { thioredoxin }\end{array}$ & $1.894317881^{a}$ & -0.65529 \\
\hline ABZJ_00028 & $\begin{array}{l}\text { lytic murein } \\
\text { transglycosylase } \\
\text { family protein }\end{array}$ & $1.296751692^{a}$ & -0.57293 \\
\hline ABZJ_01078 & hypothetical protein & -1.081092562 & -0.44448 \\
\hline ABZJ_03720 & $\begin{array}{l}\text { UDP-3-O-acyl-N- } \\
\text { acetylglucosamine } \\
\text { deacetylase }\end{array}$ & -1.144287283 & -0.48378 \\
\hline ABZJ_03859 & $\begin{array}{l}\text { putative RND type } \\
\text { efflux pump involved in } \\
\text { aminoglycoside } \\
\text { resistance (AdeT) }\end{array}$ & -1.173634714 & -0.60779 \\
\hline ABZJ_03744 & hypothetical protein & -1.296782077 & -0.60878 \\
\hline ABZJ_03737 & hypothetical protein & -1.302692756 & -1.28419 \\
\hline ABZJ_01025 & $\begin{array}{l}\text { homocysteine/ } \\
\text { selenocysteine } \\
\text { methylase }\end{array}$ & -1.337189269 & -0.71762 \\
\hline ABZJ_01219 & hypothetical protein & -1.864476303 & -0.75975 \\
\hline ABZJ_01088 & carbonic anhydrase & -1.949843631 & -0.56001 \\
\hline ABZJ_01206 & hypothetical protein & -3.281014801 & -0.4346 \\
\hline
\end{tabular}

${ }^{a}$ The result of three gene/proteins were contradictory.

\section{AUTHOR CONTRIBUTIONS}

$\mathrm{XH}$ and YY conceived and designed the study. XH, LL, YF, QS, XL, QC, KS, YJ, and HZ performed the experiments. XH and YY performed data analysis and drafted the manuscript. All authors reviewed and approved the final manuscript. 


\section{ACKNOWLEDGMENTS}

This work was supported by the National Natural Science Foundation of China $(81230039,31670135,81378158)$, the 973

\section{REFERENCES}

Antunes, L. C., Visca, P., and Towner, K. J. (2014). Acinetobacter baumannii: evolution of a global pathogen. Pathog. Dis. 71, 292-301. doi: 10.1111/2049-632X.12125

Bae, S., Kim, M. C., Park, S. J., Kim, H. S., Sung, H., Kim, M. N., et al. (2016). In vitro synergistic activity of antimicrobial agents in combination against clinical isolates of colistin-resistant Acinetobacter baumannii. Antimicrob Agents Chemother. 60, 6774-6779. doi: 10.1128/AAC.00839-16

Beceiro, A., Llobet, E., Aranda, J., Bengoechea, J. A., Doumith, M., Hornsey, M., et al. (2011). Phosphoethanolamine modification of lipid A in colistinresistant variants of Acinetobacter baumannii mediated by the pmrAB twocomponent regulatory system. Antimicrob. Agents Chemother. 55, 3370-3379. doi: 10.1128/AAC.00079-11

Beceiro, A., Moreno, A., Fernandez, N., Vallejo, J. A., Aranda, J., Adler, B., et al. (2014). Biological cost of different mechanisms of colistin resistance and their impact on virulence in Acinetobacter baumannii. Antimicrob. Agents Chemother. 58, 518-526. doi: 10.1128/AAC.01597-13

Bojkovic, J., Richie, D. L., Six, D. A., Rath, C. M., Sawyer, W. S., Hu, Q., et al. (2016). Characterization of an Acinetobacter baumannii lptD deletion strain: permeability defects and response to inhibition of lipopolysaccharide and fatty acid biosynthesis. J. Bacteriol. 198, 731-741. doi: 10.1128/JB.00639-15

Cai, Y., Chai, D., Wang, R., Liang, B., and Bai, N. (2012). Colistin resistance of Acinetobacter baumannii: clinical reports, mechanisms and antimicrobial strategies. J. Antimicrob. Chemother. 67, 1607-1615. doi: 10.1093/jac/dks084

Cheah, S. E., Johnson, M. D., Zhu, Y., Tsuji, B. T., Forrest, A., Bulitta, J. B., et al. (2016a). Polymyxin resistance in Acinetobacter baumannii: genetic mutations and transcriptomic changes in response to clinically relevant dosage regimens. Sci. Rep. 6:26233. doi: 10.1038/srep26233

Cheah, S. E., Li, J., Tsuji, B. T., Forrest, A., Bulitta, J. B., and Nation, R. L. (2016b). Colistin and polymyxin $\mathrm{B}$ dosage regimens against Acinetobacter baumannii: differences in activity and the emergence of resistance. Antimicrob. Agents Chemother. 60, 3921-3933. doi: 10.1128/AAC.02927-15

Deatherage, D. E., and Barrick, J. E. (2014). Identification of mutations in laboratory-evolved microbes from next-generation sequencing data using breseq. Methods Mol. Biol. 1151, 165-188. doi: 10.1007/978-1-4939-0554-6_12

Fang, L., Chen, Q., Shi, K., Li, X., Shi, Q., He, F., et al. (2016). Step-Wise increase in tigecycline resistance in klebsiella pneumoniae associated with Mutations in ramR, lon and rpsJ. PLoS ONE 11:e0165019. doi: 10.1371/journal.pone.0165019

Fernandez-Reyes, M., Rodriguez-Falcon, M., Chiva, C., Pachon, J., Andreu, D., and Rivas, L. (2009). The cost of resistance to colistin in Acinetobacter baumannii: a proteomic perspective. Proteomics 9, 1632-1645. doi: 10.1002/pmic.200800434

Henry, R., Crane, B., Powell, D., Deveson Lucas, D., Li, Z., Aranda, J., et al. (2015). The transcriptomic response of Acinetobacter baumannii to colistin and doripenem alone and in combination in an in vitro pharmacokinetics/pharmacodynamics model. J. Antimicrob. Chemother. 70, 1303-1313. doi: 10.1093/jac/dku536

Henry, R., Vithanage, N., Harrison, P., Seemann, T., Coutts, S., Moffatt, J. H., et al. (2012). Colistin-resistant, lipopolysaccharide-deficient Acinetobacter baumannii responds to lipopolysaccharide loss through increased expression of genes involved in the synthesis and transport of lipoproteins, phospholipids, and poly-beta-1,6-N-acetylglucosamine. Antimicrob. Agents Chemother. 56, 59-69. doi: 10.1128/AAC.05191-11
Preliminary Research Program (2014CB560707), the Natural Science Foundation of Zhejiang province, China (LY15H190004, Y16H190013) and the Zhejiang Province Medical Platform Backbone Talent Plan (2016DTA003).

Howard, A., O’Donoghue, M., Feeney, A., and Sleator, R. D. (2012). Acinetobacter baumannii: an emerging opportunistic pathogen. Virulence 3, 243-250. doi: 10.4161/viru.19700

Hua, X., Chen, Q., Li, X., and Yu, Y. (2014). Global transcriptional response of Acinetobacter baumannii to a subinhibitory concentration of tigecycline. Int. J. Antimicrob. Agents 44, 337-344. doi: 10.1016/j.ijantimicag.2014. 06.015

Li, J., Rayner, C. R., Nation, R. L., Owen, R. J., Spelman, D., Tan, K. E., et al. (2006). Heteroresistance to colistin in multidrug-resistant Acinetobacter baumannii. Antimicrob. Agents Chemother. 50, 2946-2950. doi: 10.1128/AAC. 00103-06

McCarthy, D. J., Chen, Y., and Smyth, G. K. (2012). Differential expression analysis of multifactor RNA-Seq experiments with respect to biological variation. Nucleic Acids Res. 40, 4288-4297. doi: 10.1093/nar/gks042

McClure, R., Balasubramanian, D., Sun, Y., Bobrovskyy, M., Sumby, P., Genco, C. A., et al. (2013). Computational analysis of bacterial RNA-Seq data. Nucleic Acids Res. 41:e140. doi: 10.1093/nar/gkt444

Moffatt, J. H., Harper, M., Harrison, P., Hale, J. D., Vinogradov, E., Seemann, T., et al. (2010). Colistin resistance in Acinetobacter baumannii is mediated by complete loss of lipopolysaccharide production. Antimicrob. Agents Chemother. 54, 4971-4977. doi: 10.1128/AAC.00834-10

Mu, X., Wang, N., Li, X., Shi, K., Zhou, Z., Yu, Y., et al. (2016). The Effect of Colistin Resistance-Associated Mutations on the Fitness of Acinetobacter baumannii. Front. Microbiol. 7:1715. doi: 10.3389/fmicb.2016.01715

Peleg, A. Y., Seifert, H., and Paterson, D. L. (2008). Acinetobacter baumannii: emergence of a successful pathogen. Clin. Microbiol. Rev. 21, 538-582. doi: 10.1128/CMR.00058-07

Thi Khanh Nhu, N., Riordan, D. W., Do Hoang Nhu, T., Thanh, D. P., Thwaites, G., Huong Lan, N. P., et al. (2016). The induction and identification of novel Colistin resistance mutations in Acinetobacter baumannii and their implications. Sci. Rep. 6:28291. doi: 10.1038/srep28291

Vizcaino, J. A., Csordas, A., Del-Toro, N., Dianes, J. A., Griss, J., Lavidas, I., et al. (2016). 2016 update of the PRIDE database and its related tools. Nucleic Acids Res. 44, D447-D456. doi: 10.1093/nar/gkw880

Wang, S., Yang, Y., Zhao, Y., Zhao, H., Bai, J., Chen, J., et al. (2016). Sub-MIC tylosin inhibits Streptococcus suis biofilm formation and results in differential protein expression. Front. Microbiol. 7:384. doi: 10.3389/fmicb.2016.00384

Zhou, H., Zhang, T., Yu, D., Pi, B., Yang, Q., Zhou, J., et al. (2011). Genomic analysis of the multidrug-resistant Acinetobacter baumannii strain MDRZJ06 widely spread in China. Antimicrob. Agents Chemother. 55, 4506-4512. doi: 10.1128/AAC.01134-10

Conflict of Interest Statement: The authors declare that the research was conducted in the absence of any commercial or financial relationships that could be construed as a potential conflict of interest.

Copyright (c) 2017 Hua, Liu, Fang, Shi, Li, Chen, Shi, Jiang, Zhou and Yu. This is an open-access article distributed under the terms of the Creative Commons Attribution License (CC BY). The use, distribution or reproduction in other forums is permitted, provided the original author(s) or licensor are credited and that the original publication in this journal is cited, in accordance with accepted academic practice. No use, distribution or reproduction is permitted which does not comply with these terms. 